\title{
Hybrid PET/CT and SPECT/CT Imaging: A Teaching File
}

\author{
D. Delbeke and O. Israel, eds.
}

New York, NY: Springer, 2010, 760 pages, $\$ 199$

This book is the product of 2 editors who have worked together over the past decade on projects related to technologic development, especially hybrid technology. They have felt the need to present and discuss cases that demonstrate the role of PET/CT and SPECT/CT hybrid imaging with various radiopharmaceuticals in daily practice. This book also is modeled after several books-Nuclear Medicine: A Teaching File, Practical FDG Imaging: A Teaching File, and Nuclear Cardiology and Correlative Imaging: A Teaching File - that have been useful reference resources on studies using both PET and SPECT radiopharmaceuticals.

In this comprehensive practical atlas on state-of-the art integrated PET/CT and SPECT/CT systems, in-depth cases are included that will guide and advance the professional skills of nuclear physicians and radiologists. Designed with clinical cases related to multiple clinical entities, the book can serve as a teaching manual and everyday companion for practitioners. The book is also helpful for trainees in nuclear medicine and radiology, for medical students, and for physicians whose specialties carry over into molecular imaging with radiopharmaceuticals.

The book is organized into 3 parts. Part I introduces the history and principles of hybrid imaging and discusses normal distributions, variants, pitfalls, and artifacts, with 14 case presentations. The 15 chapters of part II are devoted to clinical applications in oncology according to specific malignant tumors. The 130 cases include 9 central nervous system applications; 9 head and neck; 11 lung; 6 breast; 6 colorectal; 6 esophageal, gastric, and gastrointestinal stromal tumor; 8 hepatobiliary and pancreatic; 5 gynecologic; 7 urinary, prostate, and testis; 7 thyroid; 8 endocrine; 9 lymphoma; 7 melanoma; 7 bone; and 6 pediatric. Part III consists of 3 chapters that discuss relatively new clinical applications of hybrid imaging in the fields of cardiology, skeletal disease, and infectious disease. Each chapter begins with an introduction, followed by staging, evaluation, and therapy of the tumor, as well as a summary with references for specific clinical applications. The case presentations range from the simple to the more complex in an attempt to simulate actual clinical practice. The images, which are of PET or SPECT alone, CT alone, and fused modalities, highlight the advantages and incremental value of hybrid technology, are clear and of high quality, but include very few arrows pointing out abnormal findings. There are brief histories, findings, discussions, and final diagnoses. The references that are included at the end of each chapter are current, allowing readers to explore in greater depth the rapidly expanding volume of knowledge.

In summary, internationally recognized contributors describe the technical and clinical aspects of hybrid PET/CT and SPECT/CT in this book and provide a comprehensive review of nuclear oncology cases found in everyday practice. A wealth of illustrations reinforces the key teaching points discussed throughout. An accompanying DVD provided by Hermes Medical Solutions Inc. contains DICOM images from selected cases, is beneficial, and simulates an actual workstation used in clinical scenarios. I highly recommend this book to nuclear physicians, radiologists, trainees, and those with an interest in hybrid imaging. This book will also be helpful to referring physicians interested in learning more about how this new medical imaging can be applied to their patients.

\section{E. Edmund Kim}

M.D. Anderson Cancer Center

1515 Holcombe Blvd., Unit 1264

Houston, TX 77030

E-mail:ekim@di.mdacc.tmc.edu

COPYRIGHT (C) 2010 by the Society of Nuclear Medicine, Inc.

DOI: $10.2967 /$ jnumed. 110.078923 